\title{
Barefoot running does not affect simple reaction time: An exploratory study
}

\author{
Nicholas J Snow ${ }^{1}$, Jason F L Blair ${ }^{2}$, Graham Z MacDonald ${ }^{3}$, Jeannette M Byrne ${ }^{\text {Corresp.., }}$, $^{\text {, Fabien A Basset }}{ }^{\text {Corresp. }} 2$ \\ 1 Faculty of Medicine, Memorial University of Newfoundland, St. John's, Newfoundland and Labrador, Canada \\ 2 School of Human Kinetics and Recreation, Memorial University of Newfoundland, St. John's, Newfoundland and Labrador, Canada \\ 3 Human Performance Laboratory, Faculty of Kinesiology, University of Calgary, Calgary, Alberta, Canada \\ Corresponding Authors: Jeannette M Byrne, Fabien A Basset \\ Email address: jmbyrne@mun.ca, fbasset@mun.ca
}

Background. Converging evidence comparing barefoot (BF) and shod (SH) running highlights differences in foot-strike patterns and somatosensory feedback, among others. Anecdotal evidence from $\mathrm{SH}$ runners attempting BF running suggests a greater attentional demand may be experienced during $\mathrm{BF}$ running. However, little work to date has examined whether there is an attentional cost of BF versus SH running.

Objective. This exploratory study aimed to examine whether an acute bout of BF running would impact simple reaction time (SRT) compared to SH running, in a sample of runners naïve to BF running.

Methods. Eight male distance runners completed SRT testing during 10 minutes of BF or SH treadmill running at $70 \%$ maximal aerobic speed $\left(17.9 \pm 1.4 \mathrm{~km} \mathrm{~h}^{-1}\right)$. To test SRT, participants were required to press a hand-held button in response to the flash of a light bulb placed in the center of their visual field. SRT was tested at 1-minute intervals during running. BF and SH conditions were completed in a pseudorandomized and counterbalanced crossover fashion. SRT was defined as the time elapsed between the light bulb flash and the button press. SRT errors were also recorded and were defined as the number of trials in which a button press was not recorded in response to the light bulb flash.

Results. Overall, SRT later in the exercise bouts showed a statistically significant increase compared to earlier $(p<0.05)$. Statistically significant increases in SRT were present at 7 minutes versus 5 minutes $(0.29 \pm 0.02 \mathrm{~s}$ vs. $0.27 \pm 0.02 \mathrm{~s}, p<0.05)$ and at 9 minutes versus 2 minutes $(0.29 \pm 0.03 \mathrm{~s}$ vs. $0.27 \pm$ $0.03 \mathrm{~s}, p<0.05)$. However, BF running did not influence this increase in SRT $(p>0.05)$ or the number of SRT errors ( $17.6 \pm 6.6$ trials vs. $17.0 \pm 13.0$ trials, $p>0.05)$.

Discussion. In a sample of distance runners naïve to BF running, there was no statistically significant difference in SRT or SRT errors during acute bouts of BF and SH running. We interpret these results to mean that BF running does not have a greater attentional cost compared to SH running during a SRT task throughout treadmill running. Literature suggests that stride-to-stride gait modulation during running may occur predominately via mechanisms that preclude conscious perception, thus potentially attenuating effects of increased somatosensory feedback experienced during BF running. Future research should explore the present experimental paradigm in a larger sample using over-ground running trials, as well as employing different tests of attention. 
1 Barefoot running does not affect simple reaction time: an exploratory study.

2 Nicholas J. Snow ${ }^{1}$, Jason F. L. Blair ${ }^{2}$, Graham Z. MacDonald ${ }^{3}$, Jeannette M. Byrne ${ }^{2}{ }^{\dagger}$, Fabien A.

3 Basset $^{2, \dagger}$

$4 \quad{ }^{1}$ Faculty of Medicine, Memorial University of Newfoundland, St. John's, NL, Canada.

$5{ }^{2}$ School of Human Kinetics and Recreation, Memorial University of Newfoundland, St. John's, 6 NL, Canada

$7{ }^{3}$ Human Performance Laboratory, Faculty of Kinesiology, University of Calgary, Calgary, AB, 8 Canada

9

$10 \uparrow$ Co-principal Investigator.

11

12 Corresponding Author:

13 Fabien Basset $^{2}$

14 Physical Education Building, Memorial University of Newfoundland, St. John's, NL, A1C 5S7

15 Email address: fbasset@mun.ca 
Abstract

17

Background. Converging evidence comparing barefoot $(\mathrm{BF})$ and shod $(\mathrm{SH})$ running highlights differences in foot-strike patterns and somatosensory feedback, among others. Anecdotal evidence from SH runners attempting BF running suggests a greater attentional demand may be experienced during BF running. However, little work to date has examined whether there is an attentional cost of BF versus SH running. Objective. This exploratory study aimed to examine whether an acute bout of BF running would impact simple reaction time (SRT) compared to SH running, in a sample of runners naïve to BF running. Methods. Eight male distance runners completed SRT testing during 10 minutes of BF or SH treadmill running at $70 \%$ maximal aerobic speed $\left(17.9 \pm 1.4 \mathrm{~km} \mathrm{~h}^{-1}\right)$. To test SRT, participants were required to press a hand-held button in response to the flash of a light bulb placed in the center of their visual field. SRT was tested at 1-minute intervals during running. BF and $\mathrm{SH}$ conditions were completed in a pseudorandomized and counterbalanced crossover fashion. SRT was defined as the time elapsed between the light bulb flash and the button press. SRT errors were also recorded and were defined as the number of trials in which a button press was not recorded in response to the light bulb flash. Results. Overall, SRT later in the exercise bouts showed a statistically significant increase compared to earlier $(p<0.05)$. Statistically significant increases in SRT were present at 7 minutes versus 5 minutes $(0.29 \pm 0.02 \mathrm{~s}$ vs. $0.27 \pm 0.02 \mathrm{~s}, p<0.05)$ and at 9 minutes versus 2 minutes $(0.29 \pm 0.03 \mathrm{~s}$ vs. $0.27 \pm 0.03 \mathrm{~s}, p<0.05)$. However, $\mathrm{BF}$ running did not influence this increase in SRT $(p>0.05)$ or the number of SRT errors $(17.6 \pm 6.6$ trials vs. $17.0 \pm 13.0$ trials, $p$ $>0.05)$. Discussion. In a sample of distance runners naïve to BF running, there was no statistically significant difference in SRT or SRT errors during acute bouts of BF and SH running. We interpret these results to mean that $\mathrm{BF}$ running does not have a greater attentional 
39 cost compared to SH running during a SRT task throughout treadmill running. Literature

40 suggests that stride-to-stride gait modulation during running may occur predominately via

41 mechanisms that preclude conscious perception, thus potentially attenuating effects of increased

42 somatosensory feedback experienced during BF running. Future research should explore the

43 present experimental paradigm in a larger sample using over-ground running trials, as well as

44 employing different tests of attention. 
45

46

47

48

49

50

51

52

53

54

55

56

57

58

59

60

61

62

63

64

65

66

67

\section{Background}

Despite a considerable amount of research focusing on footwear's role in injury

prevention (Gallant \& Pierrynowski, 2014), injury rates have remained constant over the past 40 years (Lieberman, 2012). Approximately $85 \%$ of runners experience running-related musculoskeletal injuries throughout their running career, and 30-70\% of runners are treated for these injuries annually (Nielsen, Buist, Sorensen, Lind, \& Rasmussen, 2012). This high prevalence of running-related injuries has led to investigations into the mechanisms contributing to their etiology (Hreljac, 2005), and to alternative solutions beyond the classic recommendation of a change in footwear characteristics. In this context, barefoot (BF) running has been proposed as an alternative solution (Murphy, Curry, \& Matzkin, 2013), and has gained substantial traction in the public (Hryvniak, Dicharry, \& Wilder, 2014). Indeed, recent literature describes several distinct differences between shod $(\mathrm{SH})$ and BF running. Of interest are changes in foot-strike patterns (Divert, Baur, Mornieux, Mayer, \& Belli, 2005; Lieberman et al., 2010; Squadrone \& Gallozzi, 2009), movement kinematics (Squadrone \& Gallozzi, 2009), and muscle activation (Snow, Basset, \& Byrne, 2016; von Tscharner, Goepfert, \& Nigg, 2003).

BF running has been promoted as a method to increase foot plantar sensation (Robbins, Gouw, \& Hanna, 1989; Robbins, Gouw, McClaran, \& Waked, 1993; Robbins \& Hanna, 1987), feedback that is believed to be masked during SH running (Robbins et al., 1993; Robbins, Waked, \& McClaran, 1995). Improved plantar sensory feedback associated with BF running has been suggested to modify kinematic variables (e.g., stride length, stride frequency) in a way that ultimately alters foot-strike patterns (Daoud et al., 2012; Lieberman, 2012) to reduce plantar pain and the risk of injury due to repetitive impact (e.g., during SH running with a heel-to-toe footstrike; Lieberman et al., 2010). Given running involves chronic repetitive movement, it is no 
68 surprise that kinematic differences in the running gait can greatly impact one's risk of injury

69 (Daoud et al., 2012; Hreljac, 2005). Furthermore, running is associated with a high risk of acute

70 injury due to trips, falls, and sprains (Hsu, 2012; Knobloch, Yoon, \& Vogt, 2008); thus, the

71 prevalence of both acute and chronic injuries in running demonstrate that efficient cognitive

72 processing and rapid reaction to perturbations or obstacles is imperative during running.

73 Prior work has suggested that the speed of basic information processing is a valid

74 indicator of higher cognitive function (Kail \& Salthouse, 1994; Woods, Wyma, Yund, Herron, \&

75 Reed, 2015), and that decrements in higher-order cognitive operations can be reflected by

76 diminished performance in simple tasks evaluating the speed of processing (Kail \& Salthouse,

77 1994; Woods et al., 2015). Simple reaction time (SRT) is a task frequently used to measure

78 speed of processing (Woods et al., 2015). Tests of SRT often involve making a physical response

79 (e.g., pressing a button) to the presentation of a visual stimulus (e.g., light bulb flash). SRT is

80 thus defined as minimum amount of time needed to respond to the stimulus (Woods et al., 2015).

81 Furthermore, when an SRT task is simultaneously combined with another demanding situation

82 (e.g., exercise), it causes a dual-task situation (Watanabe \& Funahashi, 2017). In the dual-task,

83 poorer performance often results in one or both tasks, relative to when they are preformed alone

84 (Watanabe \& Funahashi, 2017). This dual-task interference effect has been established as an

85 important indicator of humans' limited capacity for information processing (Watanabe \&

86 Funahashi, 2017). Past evidence has demonstrated that concurrent acute exercise can result in

87 decrements in performance on SRT tasks (Brisswalter, Arcelin, Audiffren, \& Delignières, 1997;

88 McMorris \& Keen, 1994). For example, a dual-task interference effect was observed during

89 rhythmic exercise when a non-preferred foot cadence was adopted (Brisswalter, Durand,

90 Delignieres, \& Legros, 1995; Collardeau, Brisswalter, \& Audiffren, 2001). This evidence 
91 suggests that maintaining a novel stride frequency requires considerable attention, sufficient to

92 cause participants' SRT performance to deteriorate. If a non-preferred stride frequency can alter

93 SRT, then SRT could also be compromised during BF running, which promotes greater stride

94 frequency (Divert et al., 2005) and sensory feedback that causes participants to pay more

95 attention to their foot-strike (Abernethy, Hanna, \& Plooy, 2002; Brisswalter et al., 1995; Hanson,

96 Whitaker, \& Heron, 2009). In line with this idea, a recent study suggested that BF running

97 requires a greater level of attention than $\mathrm{SH}$, due to a greater need to focus on placing footfalls

98 on the ground (Alloway, Alloway, Magyari, \& Floyd, 2016).

The literature also indicates that individuals with worse reaction time performance are prone to increased ankle instability (Konradsen \& Ravn, 1990, 1991), and may be at a greater risk of falls (Richardson, Exkner, Allet, Kim, \& Ashton-Miller, 2017) and acute lower-limb injuries such as ankle sprains (Beynnon, Renström, Alosa, Baumhauer, \& Vacek, 2001; Murphy \& Connolly, 2003; Willems et al., 2005). Thus, given the greater sensory feedback associated with BF running (Robbins et al., 1989; Robbins \& Hanna, 1987), it is possible that BF running requires a greater attentional demand relative to $\mathrm{SH}$ running, potentially leading to decrements in SRT and increased acute injury risk due to sprains, collisions, stumbles, or falls. running influenced SRT compared to SH running, in a sample of competitive distance runners naïve to BF running. We hypothesized that SRT would be increased during BF running, relative to $\mathrm{SH}$ running at a similar exercise intensity.

\section{Materials \& Methods}

113 Research at the Memorial University of Newfoundland (ethics approval number: 20130246-HK), 
114 with informed consent being gathered in accordance with the principles outlined by the

115 Declaration of Helsinki.

116 Research participants were recruited using posters distributed throughout the Memorial

117 University of Newfoundland campus athletic facilities and Physical Education Building, as well

118 as local running retail outlets in the community of St. John's, NL, Canada. A standard

119 recruitment email was also distributed to local running clubs and the Memorial University of

120 Newfoundland's varsity running team.

121 Participants

122 Inclusion criteria consisted of the following: i) male participants; ii) aged between 19 and

12330 years; iii) experienced runners, operationally defined as a function of running experience $(\geq 2$

124 years of active running training), frequency ( $\geq 4$ days of running per week), and duration $(\geq 30$

125 minutes per day spent running); iv) competitive racing experience ( $\leq 18$ minutes to complete 5

$126 \mathrm{~km} ; \leq 40$ minutes to complete $10 \mathrm{~km}) ; \mathrm{v})$ experience training at a high intensity $\left(\leq 4 \mathrm{~min} \mathrm{~km}^{-1}\right.$

127 running pace, $\geq 1$ day per week); vi) free of any chronic illnesses including cardiometabolic,

128 neurological, or psychiatric diagnoses, or neuromuscular and musculoskeletal injuries, for at

129 least 3 months; vii) active use of a minimalist running shoe $(\leq 4 \mathrm{~mm}$ heel-toe drop, measured as

130 the difference between the sole height of the heel and toe of the shoe), with greater than 3

131 months of experience and at least 3 days of use per week, for at least 30 minutes per session; and 132 viii) naïve to BF running.

133 Twenty-three participants responded to recruitment materials. Of these participants, nine 134 were eligible to participate. One participant withdrew from the study after initial enrollment, due 135 to personal time constraints. Thus, eight adult male distance runners were enrolled in the present 136 study. No a priori power calculations were performed to arrive at this sample size; rather, this 
137 convenience-sample of eight participants was examined as an exploratory study, to generate data 138 intended to inform further research on the present topic. To limit variability in SRT results, we 139 recruited male participants only, given well-documented sex differences in SRT performance 140 throughout the literature (Jain, Bansal, Kumar, \& Singh, 2015; Karia, Ghuntla, Mehta, Gokhale, 141 \& Shah, 2012; Silverman, 2006; Woods, Wyma, Yund, Herron, \& Reed, 2015). Participants

142 were all competitive runners in the general preparatory phase of their training. Participants were 143 experienced with treadmill running. Participants completed both a short- and long-form Physical 144 Activity Readiness Questionnaire (PAR-Q) (Canadian Society for Exercise Physiology (CSEP), 145 2002) to ensure that they were injury-free for at least 3 months prior to enrolling in the study, and to screen for any injuries or health conditions that would preclude their inclusion in the study. Participants were instructed to refrain from strenuous exercise at least 36 hours prior to testing (Dannecker \& Koltyn, 2014); to avoid caffeine, alcohol, drugs, or supplements at least 24 hours prior to testing; and were required to obtain at least 6 hours of sleep the night prior to each testing session.

\section{Experimental Conditions}

Each participant was subjected to both BF and SH experimental conditions in a crossover

fashion (Figure 1). For the BF running condition, rubber-gripped toe-socks (Gaiam No Slip Yoga

Socks, Gaiam Inc., Boulder, CO, USA) were used. All participants were naïve to BF running and running in the socks provided. As participants had likely not developed the appropriate responses to minimize discomforts associated with true barefoot running (Lieberman, 2012; Robbins et al., 1993), the toe-socks were used to help minimize potential abrasions or friction burns associated with true barefoot running on a treadmill (Snow et al., 2016). Previous studies have employed similar garments during assessments of BF running (Divert, et al., 2008; Snow et al., 2016). For 
160 the SH running condition, participants were required to bring in a pair of their own running shoes

161 (Snow et al., 2016), to mitigate any negative influences footwear discomfort may have on SRT

162 (Mündermann, Nigg, Humble, \& Stefanyshyn, 2003). In accordance with previous studies, these

163 running shoes were $>225 \mathrm{~g}$ in mass, had a $>5 \mathrm{~mm}$ heel-toe drop, and were with or without

164 medial arch support or impact attenuation features (Esculier, Dubois, Dionne, Leblond, \& Roy,

165 2015; Rixe, Gallo, \& Silvis, 2012). Participants did not wear minimalist footwear during the 166 experiment.

\section{Experimental Set-up and Protocol}

The experimental protocol was administered over two testing sessions separated by $\geq 48$

169

170

171

172

173

174

175

176

177

178

179

180

181

182

hours, as depicted in Figure 1. All testing sessions were conducted in the morning.

Testing session one. During this baseline session, participants' informed consent, anthropometrics (i.e., body mass, height), and demographic information were collected first (Table 1, Table 2). Participants were then familiarized with the experimental conditions and setup, before completing an incremental treadmill exercise test. All running trials were conducted on a Cybex 750T motorized treadmill (Cybex International, Inc., Medway, MA, USA) set at a constant $1 \%$ grade to account for air-resistance experienced when running outdoors (Jones \& Doust, 1996).

For familiarization, participants were instructed to run at a self-selected treadmill speed in both their standard running footwear ( $\mathrm{SH}$ running, $11.0 \pm 0.5 \mathrm{~km} \mathrm{~h}^{-1}$ ) and the toe-socks provided (BF running, $10.7 \pm 0.8 \mathrm{~km} \mathrm{~h}^{-1}$ ), for 2.5 minutes each, in random order. Heart rate (HR) was not assessed during these running trials. Furthermore, these running trials were not intended to habituate participants to the footwear conditions in advance of SRT testing, but to provide them with an expectation of how each running condition felt, to aid in their decision to either 
183 continue in the subsequent study session or withdraw. Immediately following this familiarization

184 session, exercise testing was conducted, and consisted of an incremental treadmill test to

185 exhaustion. The treadmill test was used to determine maximal $\mathrm{O}_{2}$ uptake $\left(\mathrm{VO}_{2 \max }\right)$ and maximal

186 aerobic speed (MAS), defined as the participants' running speed at $\mathrm{VO}_{2 \max }$ (Basset, Chouinard,

$187 \&$ Boulay, 2003). Participants were instructed to wear their preferred footwear for exercise

188 testing, given prior evidence that running economy is greater when participants wear shoes with

189 a higher comfort rating (Luo, Stergiou, Worobets, Nigg, \& Stefanyshyn, 2009). The incremental

190 test started at a treadmill speed of $7.0 \mathrm{~km} \mathrm{~h}^{-1}$ and was increased by $1.0 \mathrm{~km} \mathrm{~h}^{-1}$ every 2 minutes

191 until participants reached volitional exhaustion (Leger \& Boucher, 1980). To ensure participants

192 reached $\mathrm{VO}_{2 \max }$ upon volitional exhaustion (as opposed to peak $\mathrm{VO}_{2}$ ), they recovered for 5

193 minutes at walking speed prior to the treadmill speed being increased to $105 \%$ of MAS

194 (Rossiter, Kowalchuk, \& Whipp, 2006). Participants were instructed to maintain $105 \%$ of MAS

195 until they reached their limit of tolerance (Rossiter et al., 2006). All participants reached $\mathrm{VO}_{2 \max }$

196 during the treadmill test.

Exercise metabolic rate of the incremental test was recorded with an indirect calorimetry system (AEI Technologies, Inc., Pittsburgh, PA, USA). Oxygen uptake $\left(\mathrm{VO}_{2}\right)$, carbon dioxide $\left(\mathrm{VCO}_{2}\right)$, breathing frequency $(\mathrm{B} f)$, and tidal volume $\left(\mathrm{V}_{\mathrm{T}}\right)$ were continuously collected with an automated open-circuit gas analysis system using $\mathrm{O}_{2}$ and $\mathrm{CO}_{2}$ analyzers (Model S-3A and Anarad AR-400, Ametek, Pittsburgh, PA), and a pneumo-tachometer (Model S-430,

Vacumetrics/Vacumed Ltd., Ventura, CA) with a 4.2 L mixing chamber. Respiratory exchange ratio $(\mathrm{RER})$ and minute ventilation $\left(\mathrm{V}_{\mathrm{E}}\right)$ were calculated as the quotient of $\mathrm{VCO}_{2}$ on $\mathrm{VO}_{2}$ and as

204 the product of $\mathrm{B} f$ by $\mathrm{V}_{\mathrm{T}}$, respectively. Online HR data were wirelessly transmitted to the AEI 205 indirect calorimetric system with a telemetric Polar HR monitor (Polar Electro, Oy, Finland). 
206 Prior to testing, volume and gas analyzers were calibrated with a $3.0 \mathrm{~L}$ calibration syringe and

207 medically certified $\mathrm{O}_{2}$ and $\mathrm{CO}_{2}$ calibration gases that were $16 \% \mathrm{O}_{2}$ and $4 \% \mathrm{CO}_{2}$, respectfully. The

208 data were online digitalized from an A/D card to a computer for monitoring the metabolic rate

209 (AEI Metabolic System Software, AEI Technologies, Inc., Pittsburgh, PA, USA). Results of the

$210 \mathrm{VO}_{2 \max }$ testing were used to determine running speeds implemented during later experimental

211 trials.

212 Testing session two. On day two of testing participants completed two, 10-minute

213 running trials, one BF and the other SH. During each of the 10-minute trials participants' SRT

214 was tested 10 times every minute. Participants' HR was not measured during these running trials.

215 Both BF and SH running conditions were completed at 70\% of the participants' MAS, which

216 was believed to coincide with a level of physiological arousal that optimizes SRT (Brisswalter et

217 al., 2002; Collardeau et al., 2001). Past work suggests that exercise at such an intensity and

218 duration can facilitate cognitive processes during exercise (Lambourne \& Tomporowski, 2010;

219 Tomporowski, 2003). This exercise intensity was selected to emphasize the potential effect that

220 footwear condition would have on SRT, without the contaminating effect of fatigue or

221 inappropriate exercise intensity. Conditions were pseudo-randomized and counterbalanced

222 across the study sample, such that the order of BF and SH running was reversed for every other

223 participant, to prevent an order effect of running conditions on SRT performance.

SRT was defined as the time required to press a hand-held button in response to the flash

225 of a $40 \mathrm{~W}$ soft-white light bulb placed in the center of the participants' visual field. The SRT

226 device used presently was developed at the Memorial University of Newfoundland and has been

227 used in previous research examining exercise effects on SRT (Behm, Bambury, Cahill, \& Power,

228 2004). The between- and within-session reliability of SRT measurements was shown by intra- 
229 class correlation coefficients of 0.60 and 0.79 (moderate to good reliability), respectively, with

230 no statistically significant $(p>0.05)$ differences between test and re-test values (Behm et al.,

231 2004). Participants held the button apparatus in their dominant hand during all SRT procedures.

232 The apparatus was also affixed to participants' dominant wrist with a fabric-lined Velcro strap,

233 to prevent dropping. To eliminate any auditory distractions, participants wore ear-plugs along

234 with a noise-cancelling headset. To eliminate any visual distractions, barricades restricted

235 participant's peripheral field of view. Both triggering of the light bulb (SRT stimulus) and the

236 button-press (SRT response) were recorded at $2000 \mathrm{~Hz}$, sampled using a BIOPAC MP100

237 biological amplifier, and displayed using AcqKnowledge 3.9.1 software (BIOPAC Systems, Inc.,

238 Goleta, CA, USA). All SRT data were stored offline on a computer and later pre-processed using

239 AcqKnowledge and Microsoft Excel software.

240 Prior to commencing each running condition, participants completed three, 20-stimulus,

241 SRT familiarization trials while standing on the treadmill at rest (Brisswalter et al., 1997, 1995).

242 Prior to both SRT familiarization and exercise periods, participants were instructed to focus on a

243 small target just below the light bulb and to respond quickly and vigilantly to the presented

244 stimuli. Following SRT familiarization, participants warmed up for 5 minutes at their self-

245 selected treadmill speed ( $\mathrm{SH}$ running, $11.0 \pm 0.5 \mathrm{~km} \mathrm{~h}^{-1}$; BF running, $10.7 \pm 0.8 \mathrm{~km} \mathrm{~h}^{-1}$ ),

246 completed the required condition (i.e. $\mathrm{BF}$ or $\mathrm{SH}$ ), and then rested for 3 minutes prior to repeating

247 the protocol, completing the second condition. In combination with the subsequent

248 familiarization SRT trials, participants were inactive (i.e., not running) for a total of 6 minutes.

249 This time allowed for recovery of HR between running conditions (Saltin et al., 1968), with the

250 intention of not influencing cognitive performance under the subsequent condition (Lambourne

251 \& Tomporowski, 2010). During each 10-minute condition (i.e., BF and SH), SRT testing was 
252 administered in blocks of 10 SRT stimuli, delivered over the last 50 seconds of each minute, with

253 each of the stimuli separated by a random interval to prevent anticipation of subsequent trials

254 (Schmidt \& Lee, 2005). The above setup was intended to produce a dual-task effect, to examine

255 which running condition would have a greater influence on participants' SRT. To complement

256 the objective SRT information provided we also asked participants to briefly comment on their

257 experience of BF versus SH running.

258 Video footage was gathered on the right lower-limb, for both SH and BF running

259 conditions to assess stride frequency. A Sony HDR-CX430VB $30 \mathrm{~Hz}$ video camera (Sony

260 Computer Entertainment America, San Mateo, CA, USA) was positioned perpendicular to the

261 treadmill at a distance of 1.5 metres and a height of 0.75 metres. Video footage was collected at a

$26230 \mathrm{~Hz}$ frame rate, in accordance with previous literature (Macpherson, Taylor, McBain, Weston,

263 \& Spears, 2016; Nikodelis, Moscha, Metaxiotis, \& Kollias, 2011), with a total of 30 running

264 strides per participant being collected for each running condition (i.e., BF and SH). Raw video

265 data were converted to MPEG-4 using Sony PMB software (Sony Computer Entertainment

266 America, San Mateo, CA, USA) for further analysis.

\section{Data Analyses}

$\mathbf{V O}_{2 \max }$. Participants' $\mathrm{VO}_{2 \max }$ was considered the peak value in $\mathrm{O}_{2}$ uptake using a 30-

second moving window average technique. MAS was the corresponding treadmill speed at

$\mathrm{VO}_{2 \max }$ (American College of Sports Medicine (ACSM), 2013). $\mathrm{HR}_{\max }$ was defined as the peak

HR value obtained during the $\mathrm{VO}_{2 \max }$ test (ACSM, 2013).

272

Stride frequency. The Kinovea (Version 2.0) high-resolution video analysis software

273 platform (http://www.kinovea.org/) was used to determine the frame of foot-contact and toe-off

274 during each 10-second window, for each minute, throughout each 10-minute trial, for both the 
275 BF and SH running conditions (Damsted, Nielsen, \& Larsen, 2015; Padulo et al., 2015). To

276 minimize error in video interpretation, blind cross-checks were performed by two researchers

277 (NJS, JMB). Foot-contact and toe-off were used to determine stride frequency (strides $\mathrm{s}^{-1}$ ) by

278 counting the number of complete strides per 10 seconds of video data. This number was then

279 multiplied by 6 to provide the final stride frequency estimate (strides $\min ^{-1}$ ).

280 SRT. Participants' SRT was considered the time difference (in seconds) between the

281 initiation of the SRT stimulus (light bulb) and the completion of the SRT response (button press)

282 (Magill, 2011). As such, SRT encompassed participants' overall response time, which is

283 comprised of both reaction time (i.e., time between stimulus presentation and initiation of

284 response) and movement time (i.e., time between response initiation and response completion)

285 (Magill, 2011). Therefore, our SRT measure contained a global measure of both stimulus

286 detection (reaction time) and response execution (movement time) but did not distinguish

287 between them. Any SRT trial $<0.160$ seconds was considered an anticipated response and was to

288 be omitted from the data set (Brisswalter et al., 1997, 1995; Collardeau et al., 2001). However,

289 zero SRT responses met this criterion, and thus no SRT trials were omitted from the data set on

290 this basis. We also analyzed SRT errors, which consisted of instances where participants did not

291 respond to the stimulus (light bulb), or when SRT trials $>1.0$ seconds were recorded (Woods et

292 al., 2015). No SRT trials exceeded 1.0 seconds, and so SRT errors were considered only those

293 trials that were un-recorded. The number of un-recorded SRT responses were counted by the

294 AcqKnowledge software during each minute and averaged across each total running trial (BF,

$295 \mathrm{SH})$. Remaining SRT trials were averaged over each minute for their respective running

296 conditions (i.e., BF and SH running). Increasing SRT (in seconds) was indicative of an increase

297 in perceptual latency and attentional load. 


\section{Statistical Analyses}

All data were examined for normality using the Shapiro-Wilk test and visual examination

300

301

302

303

304

305

306

307

308

309

310

311

312

313

314

315

316

317

318

319

of histogram plots. Because of sensitivity to sample size variations in statistics-based normality

tests, as well as the robustness of within-subjects designs to normality violations, we used a

stringent significance level of $p<0.001$ in objective examinations of the data distributions (i.e.,

Shapiro-Wilk test) (Gamst, Meyers, \& Guarino, 2008; Mang, Snow, Wadden, Campbell, \&

Boyd, 2016).

The effect of BF versus SH running on SRT was tested using a two-way (2 levels $\times 10$

levels) repeated-measures analysis of variance (rmANOVA) with the factors Condition (BF

running, SH running) and Time (1-10 min). To determine if SRT during BF running, SH

running, or running in general was different from rest, the average SRT from the familiarization

trials, the BF and SH running conditions, and both exercise conditions combined was compared

using a one-way (4 levels) rmANOVA with the factor Condition (Familiarization, BF running,

SH running, Combined running). Post hoc pairwise comparisons were conducted when

necessary using the Bonferroni correction. Mean SRT errors (number of trials) were compared

across $\mathrm{BF}$ and $\mathrm{SH}$ running conditions using a paired samples $t$-test. Finally, average stride

frequency (strides $\mathrm{min}^{-1}$ ) was compared across conditions (BF running, $\mathrm{SH}$ running) using a paired samples $t$-test.

All results are presented as means \pm one standard deviation (SD). Statistical significance was set at $p<0.05$. Effect sizes (Cohen's $d$ ) and 95\% confidence intervals (95\%CI) of effect sizes were calculated for our primary outcome (SRT in seconds) using Microsoft Excel, and interpreted as "trivial" < 0.20; "small" 0.20-0.49; "medium" 0.50-0.79; "large" > 0.80 (Cohen, 
320 1988). All statistical analyses were conducted in SPSS (Version 20, IBM Corporation, Armonk,

321 NY, USA).

322

Results

323 Data Inspection

324

All data were deemed normally distributed based on Shapiro-Wilk statistics (SRT $W_{(8)}=$ 325 $0.774-0.990, p=0.015-0.996$; stride frequency $\left.W_{(8)}=0.876-0.912, p=0.171-0.368\right)$ and histogram plot inspection. For SRT data, Mauchly’s Test of Sphericity was not statistically significant $(p>0.05)$; thus, sphericity was assumed for interpreting the results of the rmANOVA on SRT values.

\section{Participants}

Baseline characteristics. Participants were on average $25.1 \pm 3.7$ years of age, with a

body mass, height, and body mass index (BMI) of $78.4 \pm 8.9 \mathrm{~kg}, 180.7 \pm 7.8 \mathrm{~cm}$, and $24.0 \pm 2.0$

$\mathrm{kg} \mathrm{m}^{-2}$, respectively (Table 1$)$. Their training experience ranged from 1 to 8 years $(3.1 \pm 2.1 \mathrm{yr})$, and all participants were experienced in using minimalist footwear (11.5 $\pm 11.4 \mathrm{mo})$. Participants completed an average of $8.1 \pm 3.5$ training sessions per week, including $1.9 \pm 0.8$ sessions of interval training at $>75 \% \mathrm{VO}_{2 \max }$. Total training volume was $90.0 \pm 44.7 \mathrm{~km}$ per week, and 65.3 $\pm 44.9 \%$ of training volume was completed using minimalist footwear $\left(72.3 \pm 62.0 \mathrm{~km} \mathrm{wk}^{-1}\right)$.

Average $10 \mathrm{~km}$ personal best race time (mm:ss) was 37:26 2:50. This information is detailed in 338 Table 2.

$\mathbf{V} \dot{\mathbf{O}}_{2 \max }$ testing. Baseline exercise testing results can be found in Table 1. Participants' $\mathrm{VO}_{2 \max }$ was on average $61.4 \pm 6.7 \mathrm{~mL} \mathrm{~min}^{-1} \mathrm{~kg}^{-1}$, corresponding to "excellent" fitness (ACSM, 2013). In fact, all participants achieved $\mathrm{VO}_{2 \max }$ scores in the 95th to 99th percentile based on age

342 and sex norms (ACSM, 2013). Average $\mathrm{HR}_{\max }$ was $191 \pm 4 \mathrm{bpm}(96.5 \pm 6.5 \%$ age-predicted 
$343 \mathrm{HR}_{\max }$ ). Mean maximal aerobic speed (MAS) was $17.9 \pm 1.3 \mathrm{~km} \mathrm{~h}^{-1}$, while $70 \%$ MAS (for BF

344 and SH running SRT trials) was $12.5 \pm 0.9 \mathrm{~km} \mathrm{~h}^{-1}$.

345 SRT

SRT results from BF running, $\mathrm{SH}$ running, and combined across conditions are shown in

347 Figure 2. A statistically significant main effect of Time $\left(F_{(9,63)}=3.097, p=0.004\right)$ was present

348 when assessing SRT. Statistically significant increases in SRT were present at 7 minutes relative

to 5 minutes $(0.29 \pm 0.02 \mathrm{~s}$ vs. $0.27 \pm 0.02 \mathrm{~s}, p<0.05, d=-0.99,95 \% \mathrm{CI}=-1.98$ to +0.09 , large

effect), and at 9 minutes relative to 2 minutes $(0.29 \pm 0.03 \mathrm{~s}$ vs. $0.27 \pm 0.03 \mathrm{~s}, p<0.05, d=-0.67$, $95 \% \mathrm{CI}=-1.63$ to +0.37 , moderate effect). There was neither a statistically significant main effect of Condition $\left(F_{(1,7)}=1.002, p=0.350\right)$ nor a statistically significant Condition $\times$ Time interaction effect $\left(F_{(9,63)}=1.233, p=0.292\right)$. Examination of effect sizes between conditions indicated that overall, BF running had a small negative effect on SRT $(d=-0.32,95 \% \mathrm{CI}=-3.29$ to +2.65$)$. The largest negative effect BF running had on SRT was at 8 minutes $(d=-0.80$, $95 \% \mathrm{CI}=-1.65$ to +0.06 , large effect; BF SRT $=0.30 \pm 0.03 \mathrm{~s}, \mathrm{SH}$ SRT $=0.27 \pm 0.03 \mathrm{~s}$ ) when compared to SH running. However, this effect was not statistically significant $(p>0.05)$.

Figure 3 illustrates average SRT values for the familiarization trials and each experimental condition, with an increase in SRT indicating a decrement in SRT performance.

When comparing the average SRT for the familiarization trial $(0.25 \pm 0.03 \mathrm{~s}), \mathrm{SH}$ running $(0.27$ $\pm 0.02)$, BF running $(0.28 \pm 0.03 \mathrm{~s})$, and combined trials across both conditions $(0.28 \pm 0.02 \mathrm{~s})$, there was no statistically significant main effect of Condition $\left(F_{(3,21)}=2.944, p=0.057\right)$. 
366 trials). There was no statistically significant difference in SRT errors across conditions $\left(t_{(7)}=\right.$ $3671.07, p=0.918)$.

Finally, seven participants reported feeling an increase in attentional demands during BF

369 relative to SH running, while one participant noted no difference. In general, seven of eight

370 participants ( $88 \%$ ) highlighted: i) a need to focus more on their footfalls to prevent

371 uncomfortable landings; ii) a perceived change in foot-strike patterns; and iii) pain or burning on

372 the plantar surface of the foot.

\section{Stride Frequency}

Stride frequency showed a statistically significant increase during BF running $(88.3 \pm 5.6$

strides $\left.\min ^{-1}\right)$, relative to $\mathrm{SH}$ running $\left(86.1 \pm 5.7\right.$ strides $\min ^{-1}, p<0.05, d=0.39,95 \% \mathrm{CI}=-0.62$ to +1.36 , small effect).

\section{Discussion}

The primary aim of the present study was to determine whether there was a difference in

SRT during acute bouts of $\mathrm{BF}$ and $\mathrm{SH}$ running, in competitive distance runners naïve to BF

running. Despite a statistically significant increase in SRT during later time-points of the

exercise bouts compared to earlier, we did not observe a statistically significant difference in

SRT across footwear conditions. We also did not find a statistically significant difference in SRT errors between conditions. However, there was a statistically significant increase in stride in attentional demands during BF relative to SH running.

SRT and Attentional Demands

Reaction time is an ecologically-relevant measure of perceptual-motor cost of running

(Schmidt \& Lee, 2005), due to the high prevalence of acute injuries sustained during running 
389 (Hsu, 2012; Knobloch et al., 2008), combined with the common nature of situations requiring 390 reactions to extrinsic stimuli (Magill, 2011). In some instances, individuals must react quickly 391 and suddenly to an unexpected stimulus to avoid injury, making it imperative to avoid any threat 392 to reaction time performance (Magill, 2011; Schmidt \& Lee, 2005). On this basis, increased SRT 393 has been linked to a possible increased injury risk during running, due to falls and sprains 394 (Beynnon et al., 2001; Konradsen \& Ravn, 1990, 1991; Murphy \& Connolly, 2003; Richardson 395 et al., 2017; Willems et al., 2005). Past work highlights that runners need to pay more attention to their foot-strikes during BF running (Alloway et al., 2016), and to alter their running

397 kinematics to avoid noxious plantar stimuli (Lieberman et al., 2010). Consequently, we hypothesized that BF running would produce a detrimental effect on SRT performance, with reference to SH running; yet we observed no statistically significant effect of BF running on SRT or SRT errors.

In the present study, participants anecdotally reported an increase in attentional demands during BF relative to SH running, noting: i) a need to focus more on their footfalls to prevent uncomfortable landings; ii) a perceived change in foot-strike patterns, particularly during the latter minutes of BF running; and iii) pain or burning on the plantar surface of the foot. A recent study directly comparing $\mathrm{BF}$ and $\mathrm{SH}$ running trials on an indoor track showed that runners had to pay greater attention to their foot-strikes during the BF condition, as evidenced by greater working memory when stepping on targets during running (Alloway et al., 2016). This observation is supported by work that has indicated that SRT performance is decreased in the presence of externally applied cutaneous stimulation (Hanson et al., 2009). When considering the concept of dual-task interference, which emphasizes participants' limited attentional capacity (Watanabe \& Funahashi, 2017), it could be expected that cognitive task performance would 
412 suffer in the presence of an attentionally demanding procedure such as BF running. Therefore, at

413 the same relative intensity, it is possible BF running does not have any additional attentional

414 demand compared to SH running. It is also plausible that by providing participants with rubber-

415 gripped toe socks for the BF running condition, plantar sensory feedback was masked relative to

416 a true BF running condition, introducing a potential confound to our experiment. However, given

417 runners' subjective comments, we consider this possibility unlikely.

418 Stride Frequency

419 The statistically significant increase in stride frequency with BF running may reflect

420 kinematic differences between BF and SH running (Divert et al., 2005; Ekizos, Santuz, \&

421 Arampatzis, 2017). Increased stride frequency could be the result of increased somatosensory

422 feedback present during BF running and intended to avoid painful foot-strikes (Hsu, 2012;

423 Lieberman, 2012). Indeed, increased sensory feedback during BF running can alter foot-strike

424 patterns, for instance by modulating ankle coordination prior to foot-strike (Kurz \& Stergiou,

425 2004), reducing ground reaction forces (Lieberman et al., 2010), and increasing and decreasing

426 stride frequency and contact time, respectively (Ekizos et al., 2017; Kurz \& Stergiou, 2004).

427 During SH running, this protective feedback is believed to be impaired (Robbins et al., 1995).

428 We anticipated that such differences in somatosensory feedback would have a limiting effect on

429 attentional capacity, reflected by an increase in SRT. Yet, despite the statistically significant

430 increase in stride frequency during BF running, SRT did not change.

\section{Possible Explanations}

432 Despite anecdotal reports and past research suggesting a potential attentional difference

433 between $\mathrm{BF}$ and $\mathrm{SH}$ footwear conditions, we did not observe such an effect. There is evidence in

434 support of our finding, that SRT was not different during BF versus SH running. For example, 
435 Klint et al. (2008) showed that proprioceptive and cutaneous feedback from the foot and leg can

436 modulate stepping patterns and increase variation in kinematics during BF locomotion,

437 independent of higher processing. They concluded that higher centers are likely reserved for

438 more complex movements. Others have intimated that gait involves the integration of spinal

439 activity, afferent sensory information, and efferent motor commands from the primary motor

440 cortex and pyramidal tract, with reactions to external perturbations being integrated at the

441 cortical or at the spinal level, depending on the nature of the perturbation (Nielsen, 2003).

442 Nielsen (2003) proposes that concomitant spinal and supraspinal regulation of gait allows for

443 more flexible integration of sensory (e.g., visual, somatosensory) and motor information online.

444 This is likely why neural interactions governing gait modulation are rapid, allowing stride-to-

445 stride variation in stepping patterns (Klint et al., 2008; Nigg \& Wakeling, 2001).

446 Greater variation of stride kinematics during BF running might therefore be associated

447 with injury prevention and pain reduction, due to a reduction in repeated impact (Lieberman et

448 al., 2010). However, if the increased afferent feedback experienced during BF running does not

449 undergo higher processing (Klint et al., 2008), then runners would be at not greater risk of acute

450 injuries due to stumbles or falls (Beynnon et al., 2001; Konradsen \& Ravn, 1990, 1991; D. F.

451 Murphy \& Connolly, 2003; Richardson et al., 2017; Willems et al., 2005). This is supported by

452 our finding that BF running did not have a statistically significant effect on SRT. Although

453 speculative in nature, this lack of conscious processing of somatosensory and proprioceptive

454 information would serve a protective role for persons engaging in BF running.

\section{Methodological Considerations and Future Directions}

There are a few noteworthy methodological considerations in the current work which

457 could have influenced our findings. Throughout the course of prolonged exercise fatigue can 
458 negatively influence corticospinal and neuromuscular output (Meardon, Hamill, \& Derrick,

459 2011; Ross, Middleton, Shave, George, \& Nowicky, 2007), and consequently reduce perceptual-

460 motor performance (Brisswalter et al., 2002). The present results support existing evidence in

461 that SRT performance tended to increase towards the end of the exercise bout, a result that has

462 been previously reported in the literature (Brisswalter et al., 1995; Brisswalter et al., 2002;

463 Collardeau et al., 2001; McMorris \& Keen, 1994). However, this was a short and moderately-

464 intense bout of steady-state exercise (ACSM, 2013), with an appropriately-timed rest period

465 (Saltin et al., 1968); so, fatigue was not likely a major contributor to decreased performance

466 (Lambourne \& Tomporowski, 2010; Tomporowski, 2003). Indeed, the exercise duration and

467 intensity were chosen to coincide with a level of physiological arousal that optimizes SRT

468 (Brisswalter et al., 2002; Collardeau et al., 2001; Lambourne \& Tomporowski, 2010;

469 Tomporowski, 2003). However, given we did not measure HR or perceived exertion throughout

470 SRT testing, it is possible that participants' level of exertion during exercise was greater than the

471 exercise intensity prescribed. Further work should examine HR and perceived exertion during

472 exercise and SRT testing. Aside from exercise intensity, the decrease in performance observed

473 may have been a result of the mode of exercise (i.e., treadmill exercise), as compared to exercise

474 intensity or fatigue (Lambourne \& Tomporowski, 2010). In other words, given the dual-task

475 nature of the present experiment, SRT performance may simply have suffered in response to

476 participants' avoiding falling off the treadmill. Nevertheless, there was no statistically significant

477 difference in SRT during resting familiarization trials compared to exercise. In addition, without

478 the use of treadmill running, it would not have been possible for us to employ the present SRT

479 task. 
Secondly, our measure of SRT was unable to decompose participants' overall response

481

482

483

484

485

486

487

488

489

490

491

492

493

494

495

496

497

498

499

500

501

502

time, into its constituent components of reaction time (i.e., time between stimulus presentation

and initiation of response), which measures stimulus detection; and movement time (i.e., time

between response initiation and response completion), which measures response execution

(Magill, 2011). Past work has shown that acute exercise preferentially influences movement time over reaction time in SRT tasks (Beyer, Sage, Staines, Middleton, \& McIlroy, 2017; Davranche, Burle, Audiffren, \& Hasbroucq, 2005, 2006), indicating that exercise-induced changes in response time are related more to faster movement execution than changes in cognitive function (Beyer et al., 2017). Thus, to elucidate the cognitive influence of BF running it would be prudent for further work to examine a greater number of dimensions of task performance, including separating reaction and movement times. Similarly, examining more complex cognitive tasks (e.g., discrimination RT) may better discern the cognitive impacts of BF running, as opposed to SRT which simply examines speed of information processing (Alloway et al., 2016; Beyer et al., 2017). Finally, it is conceivable that the small sample size in this exploratory study may have threatened the validity of the observed results. Likewise, by including a sample of male runners exclusively, our findings may not be generalizable to female athletes. Consequently, future work will benefit from examining a larger sample of runners, including both females and males, and recreational runners who have no experience using minimalist footwear. Subsequent investigations should also sample a larger number of SRT trials over a longer time-period, with measurements of physiological and perceived exertion.

\section{Conclusions}

In the present exploratory study, an acute bout of BF versus SH running did not impact SRT. It is possible that increased afferent feedback during BF running (Kurz \& Stergiou, 2004; 
503 Robbins et al., 1993) is responded to in subcortical regions or transcortical reflex pathways

504 (Nielsen, 2003), without affecting the attentional requirements of the task. Additionally, this may

505 be the case only for simple tasks such as SRT. Alternatively, it is possible that our small sample

506 size did not have sufficient power to reveal a significance across BF and SH running conditions.

507 Nevertheless, the present results suggest that although differences in running kinematics across

508 BF and SH running may lead to differences in musculoskeletal injuries (Daoud et al., 2012;

509 Hreljac, 2005), it is not likely that BF running will impact runners' risk of attention-related acute

510 injuries such as trips or falls (Hsu, 2012; Knobloch, Yoon, \& Vogt, 2008). Future work should

511 examine whether more complex perceptual-motor tasks and more sensitive outcomes will be

512 affected by BF versus SH running. Further efforts should also examine whether the present

513 observations will emerge in larger sample of runners. Finally, it is prudent to examine whether

514 changes in SRT will manifest when runners are performing over-ground on a stable running

515 surface, as opposed to during treadmill running. 
517 The current study received no external funding support, and the authors have no conflicts 518 of interest to declare. We wish to sincerely thank Mr. Blaise Dubois for his tremendous support

519 during experimental planning, Dr. Normand Teasdale for his generous assistance in reviewing

520 the original manuscript before its initial submission, and Dr. Thamir Alkanani for his technical 521 contributions. 


\section{References}

523 Abernethy, B., Hanna, A., \& Plooy, A. (2002). The attentional demands of preferred and non-

524 preferred gait patterns. Gait and Posture, 15(3), 256-265. https://doi.org/10.1016/S0966-

$525 \quad 6362(01) 00195-3$

526 Alloway, R. G., Alloway, T. P., Magyari, P. M., \& Floyd, S. (2016). An exploratory study

527 investigating the effects of barefoot running on working memory. Perceptual and Motor

$528 \quad$ Skills, 122(2), 432-443. https://doi.org/10.1177/0031512516640391

529 American College of Sports Medicine (ACSM). (2013). ACSM's Guidelines for Exercise Testing

530 and Prescription. (9th ed.). Baltimore, MD: Lippincott Williams and Wilkins.

531 Basset, F. A., Chouinard, R., \& Boulay, M. R. (2003). Training profile counts for time-to-

532 exhaustion performance. Canadian Journal of Applied Physiology = Revue Canadienne de

533 Physiologie Appliquee, 28(4), 654-666.

534 Behm, D. G., Bambury, A., Cahill, F., \& Power, K. (2004). Effect of acute static stretching on 535 force, balance, reaction time, and movement time. Medicine and Science in Sports and Exercise, 36(8), 1397-1402. https://doi.org/10.1249/01.MSS.0000135788.23012.5F

537

538

539

540

541

542

543

544

Beyer, K. B., Sage, M. D., Staines, W. R., Middleton, L. E., \& McIlroy, W. E. (2017). A single aerobic exercise session accelerates movement execution but not central processing. Neuroscience, 346, 149-159. https://doi.org/10.1016/j.neuroscience.2017.01.020

Beynnon, B. D., Renström, P. a, Alosa, D. M., Baumhauer, J. F., \& Vacek, P. M. (2001). Ankle ligament injury risk factors: a prospective study of college athletes. Journal of Orthopaedic Research: Official Publication of the Orthopaedic Research Society, 19(2), 213-220. https://doi.org/10.1016/S0736-0266(00)90004-4

Brisswalter, J., Arcelin, R., Audiffren, M., \& Delignières, D. (1997). Influence of physical 
exercise on simple reaction time: effect of physical fitness. Perceptual and Motor Skills, 85(3 Pt 1), 1019-27. https://doi.org/10.2466/pms.1997.85.3.1019

547

Brisswalter, J., Collardeau, M., \& René, A. (2002). Effects of acute physical exercise characteristics on cognitive performance. Sports Medicine (Auckland, N.Z.), 32(9), 555566.

Brisswalter, J., Durand, M., Delignieres, D., \& Legros, P. (1995). Optimal and non-optimal demand in a dual task of pedalling and simple reaction time: effects on energy expenditure and cognitive performance. Journal of Human Movement Studies, 29, 15-34.

Canadian Society for Exercise Physiology (CSEP). (2002). Physical activity readiness questionnaire - PAR-Q. Retrieved from http://www.csep.ca/cmfiles/publications/parq/parq.pdf

Cohen, J. (1988). Statistical power analysis for the behavioral sciences. (2nd ed.). Hillsdale, NJ: Lawrence Earlbaum Associates.

Collardeau, M., Brisswalter, J., \& Audiffren, M. (2001). Effects of a prolonged run on simple reaction time of well trained runners. Perceptual and Motor Skills, 93(3), 679-689. https://doi.org/10.2466/PMS.93.7.679-689

Damsted, C., Nielsen, R. O., \& Larsen, L. H. (2015). Reliability of video-based quantification of the knee- and hip ankle at foot strike during running. Int J Sports Phys Ther, 10(2), 147-54.

Dannecker, E. A., \& Koltyn, K. F. (2014). Pain during and within hours after exercise in healthy adults. Sports Medicine, 44(7), 921-942. https://doi.org/10.1007/s40279-014-0172-z

Daoud, A. I., Geissler, G. J., Wang, F., Saretsky, J., Daoud, Y. A., \& Lieberman, D. E. (2012). Foot strike and injury rates in endurance runners: a retrospective study. Medicine and Science in Sports and Exercise, 44(7), 1325-1334. 
568 Davranche, K., Burle, B., Audiffren, M., \& Hasbroucq, T. (2005). Information processing during

569 physical exercise: A chronometric and electromyographic study. Experimental Brain

$570 \quad$ Research, 165(4), 532-540. https://doi.org/10.1007/s00221-005-2331-9

571 Davranche, K., Burle, B., Audiffren, M., \& Hasbroucq, T. (2006). Physical exercise facilitates

572 motor processes in simple reaction time performance: An electromyographic analysis.

$573 \quad$ Neuroscience Letters, 396(1), 54-56. https://doi.org/10.1016/j.neulet.2005.11.008

574 Divert, C., Baur, H., Mornieux, G., Mayer, F., \& Belli, A. (2005). Stiffness adaptations in shod

575 running. Journal of Applied Biomechanics, 21(4), 311-321.

576 Divert, C., Mornieux, G., Freychat, P., Baly, L., Mayer, F., Belli, A. (2008). Barefoot-shod

577 running differences: shoe or mass effect? International Journal of Sports Medicine, 29, 512-

$578 \quad$ 518. https://doi.org/10.1055/s-2007-989233

579 Ekizos, A., Santuz, A., \& Arampatzis, A. (2017). Transition from shod to barefoot alters

580 dynamic stability during running. Gait \& Posture, 56(April), 31-36.

581 https://doi.org/https://doi.org/10.1016/j.gaitpost.2017.04.035

582 Esculier, J.-F., Dubois, B., Dionne, C. E., Leblond, J., \& Roy, J. S. (2015). A consensus

583 definition and rating scale for minimalist shoes. Journal of Foot and Ankle Research, 8(1),

584 42. https://doi.org/10.1186/s13047-015-0094-5

585 Gallant, J. L., \& Pierrynowski, M. R. (2014). A theoretical perspective on running-related 586 injuries. Journal of the American Podiatric Medical Association, 104(2), 211-220.

587 Gamst, G., Meyers, L. S., \& Guarino, A. J. (2008). ANOVA Assumptions. In Analysis of 588 Variance Designs (pp. 49-84). New York, NY: Cambridge University Press.

589 Hanson, J. V. M., Whitaker, D., \& Heron, J. (2009). Preferential processing of tactile events

$590 \quad$ under conditions of divided attention. Neuroreport, 20(15), 1392-1396. 
592

593

594

595

596

597

598

599

600

601

602

603

604

605

606

607

608

609

610

611

612

613

Hreljac, A. (2005). Etiology, prevention, and early intervention of overuse injuries in runners: a biomechanical perspective. Physical Medicine and Rehabilitation Clinics of North America, 16(3), 651-67, vi.

Hryvniak, D., Dicharry, J., \& Wilder, R. (2014). Barefoot running survey: Evidence from the field. Journal of Sport and Health Science, 3(2), 131-136. https://doi.org/10.1016/j.jshs.2014.03.008

Hsu, A. R. (2012). Topical review: Barefoot running. Foot \& Ankle International, 33(9), 787794. https://doi.org/10.3113/FAI.2012.0787

Jain, A., Bansal, R., Kumar, A., \& Singh, K. (2015). A comparative study of visual and auditory reaction times on the basis of gender and physical activity levels of medical first year students. International Journal of Applied and Basic Medical Research, 5(2), 124. https://doi.org/10.4103/2229-516X.157168

Jones, A. M., \& Doust, J. H. (1996). A 1\% treadmill grade most accurately reflects the energetic cost of outdoor running. Journal of Sports Sciences, 14(4), 321-327.

Kail, R., \& Salthouse, T. A. (1994). Processing speed as a mental capacity. Acta Psychologica (Amst), 86, 199-225.

Karia, R., Ghuntla, T., Mehta, H., Gokhale, P., \& Shah, C. (2012). Effect of gender difference on visual reaction time: A study on medical students of Bhavnagar region. IOSR Journal of Pharmacy, 2(3), 452-454.

Klint, R. A. F., Nielsen, J. B., Cole, J., Sinkjaer, T., \& Grey, M. J. (2008). Within-step modulation of leg muscle activity by afferent feedback in human walking. The Journal of Physiology, 586(Pt 19), 4643-4648. https://doi.org/10.1113/jphysiol.2008.155002 
614 Knobloch, K., Yoon, U., \& Vogt, P. M. (2008). Acute and overuse injuries correlated to hours of 615 training in master running athletes. Foot \& Ankle International / American Orthopaedic 616 Foot and Ankle Society [and] Swiss Foot and Ankle Society, 29(7), 671-676.

617 https://doi.org/10.3113/FAI.2008.0671

618 Konradsen, L., \& Ravn, J. B. (1990). Ankle instability caused by prolonged peroneal reaction 619 time. Acta Orthopaedica Scandinavica, 61(15), 388-390.

620 Konradsen, L., \& Ravn, J. B. (1991). Prolonged peroneal reaction time in ankle instability. 621 International Journal of Sports Medicine, 12(3), 290-292. https://doi.org/10.1055/s-20071024683

Kurz, M. J., \& Stergiou, N. (2004). Does footwear affect ankle coordination strategies? Journal of the American Podiatric Medical Association, 94(1), 53-58.

Lambourne, K., \& Tomporowski, P. (2010). The effect of exercise-induced arousal on cognitive task performance: a meta-regression analysis. Brain Research, 1341, 12-24. https://doi.org/10.1016/j.brainres.2010.03.091

Leger, L., \& Boucher, R. (1980). An indirect continuous running multistage field test: the

629 Universite de Montreal track test. Canadian Journal of Applied Sport sciences.Journal 630 Canadien Des Sciences Appliquees Au Sport, 5(2), 77-84.

631 Lieberman, D. E. (2012). What we can learn about running from barefoot running: an 632 evolutionary medical perspective. Exercise and Sport Sciences Reviews, 40(2), 63-72.

Lieberman, D. E., Venkadesan, M., Werbel, W. A., Daoud, A. I., D’Andrea, S., Davis, I. S., ... 634 Pitsiladis, Y. (2010). Foot strike patterns and collision forces in habitually barefoot versus shod runners. Nature, 463(7280), 531-535.

636 Luo, G., Stergiou, P., Worobets, J., Nigg, B., \& Stefanyshyn, D. (2009). Improved footwear 

comfort reduces oxygen consumption during running. Footwear Science, 1, 25-29.

638 Macpherson, T. W., Taylor, J., McBain, T., Weston, M., \& Spears, I. R. (2016). Real-time 639 measurement of pelvis and trunk kinematics during treadmill locomotion using a low-cost 640 depth-sensing camera: A concurrent validity study. Journal of Biomechanics, 49(3), 474641 478. https://doi.org/10.1016/j.jbiomech.2015.12.008

Magill, R. A. (2011). Motor Learning and Control: Concepts and Applications (9th ed.). New 643 York, NY: McGraw-Hill.

Mang, C. S., Snow, N. J., Wadden, K. P., Campbell, K. L., \& Boyd, L. A. (2016). High-intensity aerobic exercise enhances motor memory retrieval. Medicine and Science in Sports and Exercise, 48(12). https://doi.org/10.1249/MSS.0000000000001040

McMorris, T., \& Keen, P. (1994). Effect of exercise on simple reaction times of recreational athletes. Perceptual and Motor Skills, 78, 123-130.

Meardon, S. A., Hamill, J., \& Derrick, T. R. (2011). Running injury and stride time variability over a prolonged run. Gait \& Posture, 33(1), 36-40.

Mündermann, A., Nigg, B. M., Humble, R. N., \& Stefanyshyn, D. J. (2003). Orthotic comfort is related to kinematics, kinetics, and EMG in recreational runners. Medicine and Science in Sports and Exercise, 35(24), 1710-1719. https://doi.org/10.1249/01.MSS.0000089352.47259.CA

Murphy, D. F., \& Connolly, A. J. (2003). Risk factors for lower extremity injury: a review of the literature. Br J Sports Med, 37, 13-29. https://doi.org/10.1136/bjsm.37.1.13

657 Murphy, K., Curry, E. J., \& Matzkin, E. G. (2013). Barefoot running: does it prevent injuries? $658 \quad$ Sports Medicine (Auckland, N.Z.), 43(11), 1131-1138.

659 Nielsen, J. B. (2003). How we walk: central control of muscle activity during human walking. 
660

661

662

663

664

665

666

667

668

669

670

671

672

673

674

675

676

677

678

679

680

681

682

The Neuroscientist : A Review Journal Bringing Neurobiology, Neurology and psychiatryNeuroscientist, 9(3), 195-204.

Nielsen, R. O., Buist, I., Sorensen, H., Lind, M., \& Rasmussen, S. (2012). Training errors and running related injuries: a systematic review. International Journal of Sports Physical Therapy, 7(1), 58-75.

Nigg, B. M., Wakeling, J. M. (2001). Impact forces and muscle tuning: A new paradigm. Exercise and Sport Sciences Reviews, 29(1), 37-41.

Nikodelis, T., Moscha, D., Metaxiotis, D., \& Kollias, I. (2011). Commercial video frame rates can produce reliable results for both normal and CP spastic gait's spatiotemporal, angular, and linear displacement variables. Journal of Applied Biomechanics, 27(3), 266-271.

Padulo, J., Vando, S., Chamari, K., Chaouachi, A., Bagno, D., \& Pizzolato, F. (2015). Validity of the MarkWiiR for kinematic analysis during walking and running gaits. Biology of Sport, 32(1), 53-58. https://doi.org/10.5604/20831862.1127282

Richardson, J. K., Exkner, J. T., Allet, L., Kim, H., \& Ashton-Miller, J. (2017). Complex and simple clinical reaction times are associated with gait, balance, and major fall injury in older subjects with diabetic peripheral neuropathy. American Journal of Physical Medicine \& Rehabilitation, 96(1), 8-16. https://doi.org/doi:10.1097/PHM.0000000000000604.

Rixe, J. A., Gallo, R. A., \& Silvis, M. L. (2012). The barefoot debate: can minimalist shoes reduce running-related injuries? Current Sports Medicine Reports, 11(3), 160-165.

Robbins, S. E., Gouw, G. J., \& Hanna, A. M. (1989). Running-related injury prevention through innate impact-moderating behavior. Medicine and Science in Sports and Exercise, 21(2), $130-139$.

Robbins, S. E., Gouw, G. J., McClaran, J., \& Waked, E. (1993). Protective sensation of the 
plantar aspect of the foot. Foot and Ankle, 14(6), 347-352.

684

685

686

687

688

689

690

691

692

693

694

695

696

697

698

699

700

701

702

703

704

705

Robbins, S. E., \& Hanna, A. M. (1987). Running-related injury prevention through barefoot adaptations. Medicine and Science in Sports and Exercise, 19(2), 148-156.

Robbins, S., Waked, E., \& McClaran, J. (1995). Proprioception and stability: foot position awareness as a function of age and footwear. Age and Ageing, 24, 67-72. https://doi.org/10.1093/ageing/24.1.67

Ross, E. Z., Middleton, N., Shave, R., George, K., \& Nowicky, A. (2007). Corticomotor excitability contributes to neuromuscular fatigue following marathon running in man. Experimental Physiology, 92(2), 417-26. https://doi.org/10.1113/expphysiol.2006.035972

Rossiter, H. B., Kowalchuk, J. M., \& Whipp, B. J. (2006). A test to establish maximum O2 uptake despite no plateau in the $\mathrm{O} 2$ uptake response to ramp incremental exercise. Journal of Applied Physiology (Bethesda, Md.: 1985), 100(3), 764-770.

Saltin, B., Blomqvist, G., Mitchell, J. H., Johnson, R. L. J., Wildenthal, K., \& Chapman, C. B. (1968). Response to exercise after bed rest and after training. Circulation, 38(5 Suppl), VII1-78.

Schmidt, R. A., \& Lee, T. D. (2005). Motor Control and Learning: A Behavioral Emphasis. Champaign, IL, USA: Human Kinetics.

Silverman, I. W. (2006). Sex differences in simple visual reaction time: A historical metaanalysis. Sex Roles, 54(1-2), 57-68. https://doi.org/10.1007/s11199-006-8869-6

Snow, N. J., Basset, F. A., \& Byrne, J. (2016). An acute bout of barefoot running alters lowerlimb muscle activation for minimalist shoe users. International Journal of Sports Medicine, 37(5), 382-387. https://doi.org/10.1055/s-0035-1565140.

Squadrone, R., \& Gallozzi, C. (2009). Biomechanical and physiological comparison of barefoot 
706

707

708

709

710

711

712

713

714

715

716

717

718

719

720

721

722

723

724

and two shod conditions in experienced barefoot runners. The Journal of Sports Medicine and Physical Fitness, 49(1), 6-13.

Tomporowski, P. D. (2003). Effects of acute bouts of exercise on cognition. Acta Psychologica, $112(3), 297-324$.

von Tscharner, V., Goepfert, B., \& Nigg, B. M. (2003). Changes in EMG signals for the muscle tibialis anterior while running barefoot or with shoes resolved by non-linearly scaled wavelets. Journal of Biomechanics, 36(8), 1169-1176.

Watanabe, K., \& Funahashi, S. (2017). Toward an understanding of the neural mechanisms underlying dual-task performance: Contribution of comparative approaches using animal models. Neuroscience \& Biobehavioral Reviews, 84(February 2017), 12-28. https://doi.org/10.1016/j.neubiorev.2017.08.008

Willems, T. M., Witvrouw, W., Delbaere, K., Mahieu, N., De Bourdeaudhuij, I., \& De Clercq, D. (2005). Intrinsic risk factors for inversion ankle sprains in male subjects: a prospective study. American Journal of Sports Medicine, 33(3), 415-423. https://doi.org/10.1177/0363546504268137

Woods, D. L., Wyma, J. M., Yund, E. W., Herron, T. J., \& Reed, B. (2015). Factors influencing the latency of simple reaction time. Frontiers in Human Neuroscience, 9(March), 1-12. https://doi.org/10.3389/fnhum.2015.00131

(1) 
726 Figure 1. Schematic of experimental protocol for simple reaction time (SRT) testing.

727 Figure 2. Mean SRT from $1 \mathrm{~min}$ to $10 \mathrm{~min}$ during barefoot and shod running conditions, as well

728 as averaged across exercise conditions.

729 Figure 3. Average SRT values for familiarization, barefoot running, and shod running trials, as

730 well as averaged across exercise conditions.

731 Figure 4. Average SRT errors across barefoot and shod running trials. 
Figure 1. Schematic of experimental protocol for simple reaction time (SRT) testing.

734

735

736

737

738

739

740

741

742

743

744

745

746

747

748

749

750

751

752

753

754

Briefly, eight participants attended the laboratory on Day 1 for informed consent, measurement of anthropometrics (i.e., body mass, height), collection of demographic information, experimental setup familiarization, and incremental running exercise test for determination of maximal $\mathrm{O}_{2}$ uptake $\left(\mathrm{VO}_{2 \max }\right)$ and maximal aerobic speed (MAS). On Day 2, participants completed SRT testing under both barefoot (BF) and shod (SH) running conditions. The order of conditions was randomized and counterbalanced across the sample. After completion of one condition, participants crossed-over into the other (black arrow). Prior to commencing either the BF or SH running SRT trials, participants completed three, 20-stimulus SRT familiarization trials while standing at rest. Both $\mathrm{SH}$ and $\mathrm{BF}$ running conditions were preceded by a 5-min warm-up at participants' self-selected treadmill speed. Participants completed both BF and SH running conditions for 10 min each at 70\% MAS. During these running trials, 10 SRT stimuli were presented every minute. A 3-min seated rest period separated conditions; SRT was not tested during warm-up or rest periods.

Figure 2. Mean SRT from 1 min to 10 min during barefoot (BF Running, black circles with solid line) and shod (SH Running, gray circles with solid line) running conditions, as well as averaged across exercise conditions (Combined, black squares with dashed line). Increasing SRT (s) indicates diminished SRT performance. A statistically significant main effect of Time $\left(F_{(9,63)}=3.097, p=0.004\right)$ was found when assessing SRT. Statistically significant increases in SRT were present at $7 \mathrm{~min}$ relative to $5 \min (0.29 \pm 0.02 \mathrm{~s}$ vs. $0.27 \pm 0.02 \mathrm{~s}, p<0.05, d=-0.99$, $95 \% \mathrm{CI}=-1.98$ to +0.09 , large effect $)$, and at $9 \mathrm{~min}$ relative to $2 \mathrm{~min}(0.29 \pm 0.03 \mathrm{~s}$ vs. $0.27 \pm$ $0.03 \mathrm{~s}, p<0.05, d=-0.67,95 \% \mathrm{CI}=-1.63$ to +0.37 , moderate effect). Vertical bars represent one 
755 SD. Asterisks $(*)$ denote statistically significant differences $(p<0.05)$ at 7 min vs. 5 min, as well 756 as at $9 \min$ vs. $2 \mathrm{~min}$.

757

758

759

760

761

762

763

764

765

766

767

Figure 3. Average SRT values for familiarization, barefoot running (BF Running), and shod running (SH Running) trials, as well as averaged across exercise conditions (Combined). When the average SRT values across familiarization trials $(0.25 \pm 0.03 \mathrm{~s})$, SH running $(0.27 \pm$ $0.02)$, BF running $(0.28 \pm 0.03 \mathrm{~s})$, and combined exercise trials $(0.28 \pm 0.02 \mathrm{~s})$ were compared using a one-way (Condition) rmANOVA, the main effect of Condition was not statistically significant $\left(F_{(3,21)}=2.944, p=0.057\right)$. Increasing SRT $(\mathrm{s})$ indicates diminished SRT performance. Vertical bars represent one SD.

Figure 4. Average SRT errors across barefoot $(\mathrm{BF})$ and shod $(\mathrm{SH})$ running trials. Absent SRT responses represented $17.6 \%$ and $17.0 \%$ of total SRT trials under the BF and SH running conditions, respectively $(17.6 \pm 6.6$ trials vs. $17.0 \pm 13.0$ trials $)$. The difference in SRT errors across conditions was no statistically $\left(t_{(7)}=1.07, p=0.918\right)$. Vertical bars represent one SD. 


\section{Figure 1}

Schematic of experimental protocol for simple reaction time (SRT) testing.

Schematic of experimental protocol for simple reaction time (SRT) testing. Briefly, eight participants attended the laboratory on Day 1 for informed consent, measurement of anthropometrics (i.e., body mass, height), collection of demographic information, experimental setup familiarization, and incremental running exercise test for determination of maximal $\mathrm{O}_{2}$ uptake $\left(\mathrm{VO}_{2 \max }\right)$ and maximal aerobic speed (MAS). On Day 2, participants completed SRT testing under both barefoot (BF) and shod (SH) running conditions. The order of conditions was randomized and counterbalanced across the sample. After completion of one condition, participants crossed-over into the other (black arrow). Prior to commencing either the BF or SH running SRT trials, participants completed three, 20-stimulus SRT familiarization trials while standing at rest. Both $\mathrm{SH}$ and $\mathrm{BF}$ running conditions were preceded by a 5-min warm-up at participants' self-selected treadmill speed. Participants completed both $\mathrm{BF}$ and $\mathrm{SH}$ running conditions for 10 min each at 70\% MAS. During these running trials, 10 SRT stimuli were presented every minute. A 3-min seated rest period separated conditions; SRT was not tested during warm-up or rest periods.

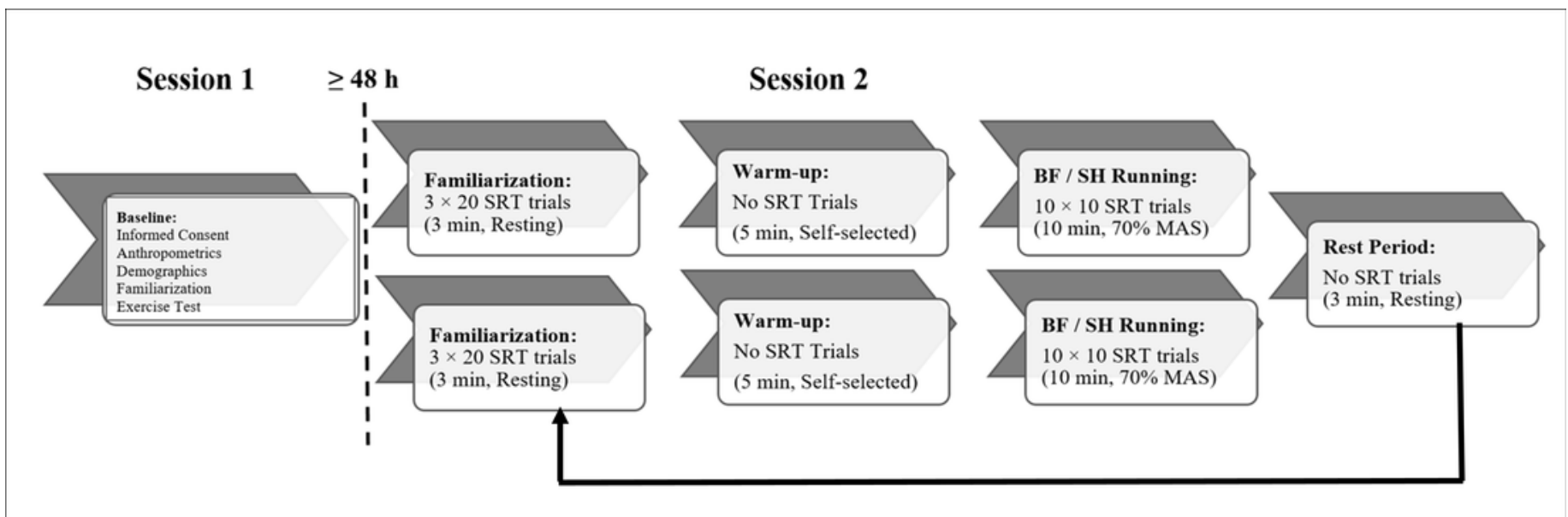




\section{Figure 2}

Mean SRT from 1 min to 10 min during barefoot (BF Running, black circles with solid line) and shod (SH Running, gray circles with solid line) running conditions, as well as averaged across exercise conditions (Combined, black squares with dashed line).

Mean SRT from 1 min to 10 min during barefoot (BF Running, black circles with solid line) and shod (SH Running, gray circles with solid line) running conditions, as well as averaged across exercise conditions (Combined, black squares with dashed line). Increasing SRT (s) indicates diminished SRT performance. A statistically significant main effect of Time $\left(F_{(9,63)}=3.097, p=\right.$ 0.004) was found when assessing SRT. Statistically significant increases in SRT were present at $7 \mathrm{~min}$ relative to $5 \mathrm{~min}(0.29 \pm 0.02 \mathrm{~s}$ vs. $0.27 \pm 0.02 \mathrm{~s}, p<0.05, d=-0.99,95 \% \mathrm{Cl}=-$ 1.98 to +0.09 , large effect), and at 9 min relative to $2 \min (0.29 \pm 0.03 \mathrm{~s}$ vs. $0.27 \pm 0.03 \mathrm{~s}, p$ $<0.05, d=-0.67,95 \% \mathrm{Cl}=-1.63$ to +0.37 , moderate effect). Vertical bars represent one SD. Asterisks $(*)$ denote statistically significant differences $(p<0.05)$ at 7 min vs. 5 min, as well as at 9 min vs. 2 min.

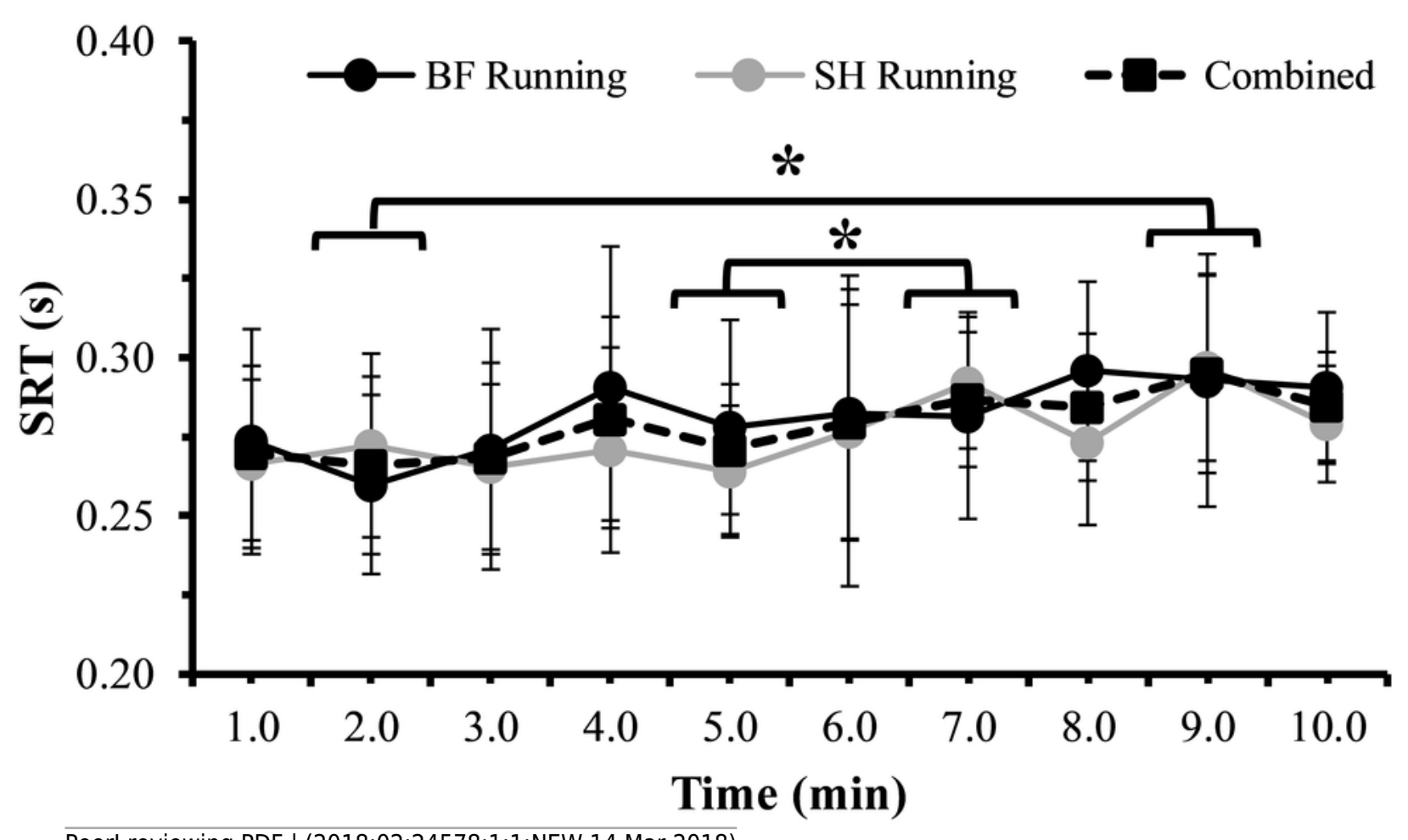




\section{Figure 3}

Average SRT values for familiarization, barefoot running (BF Running), and shod running (SH Running) trials, as well as averaged across exercise conditions (Combined).

Average SRT values for familiarization, barefoot running (BF Running), and shod running (SH Running) trials, as well as averaged across exercise conditions (Combined). When the average SRT values across familiarization trials ( $0.25 \pm 0.03 \mathrm{~s})$, SH running $(0.27 \pm 0.02)$, BF running $(0.28 \pm 0.03 \mathrm{~s})$, and combined exercise trials $(0.28 \pm 0.02 \mathrm{~s})$ were compared using a one-way (Condition) rmANOVA, the main effect of Condition was not statistically significant $\left(F_{(3,21)}=2.944, p=0.057\right)$. Increasing SRT $(\mathrm{s})$ indicates diminished SRT performance. Vertical bars represent one SD.

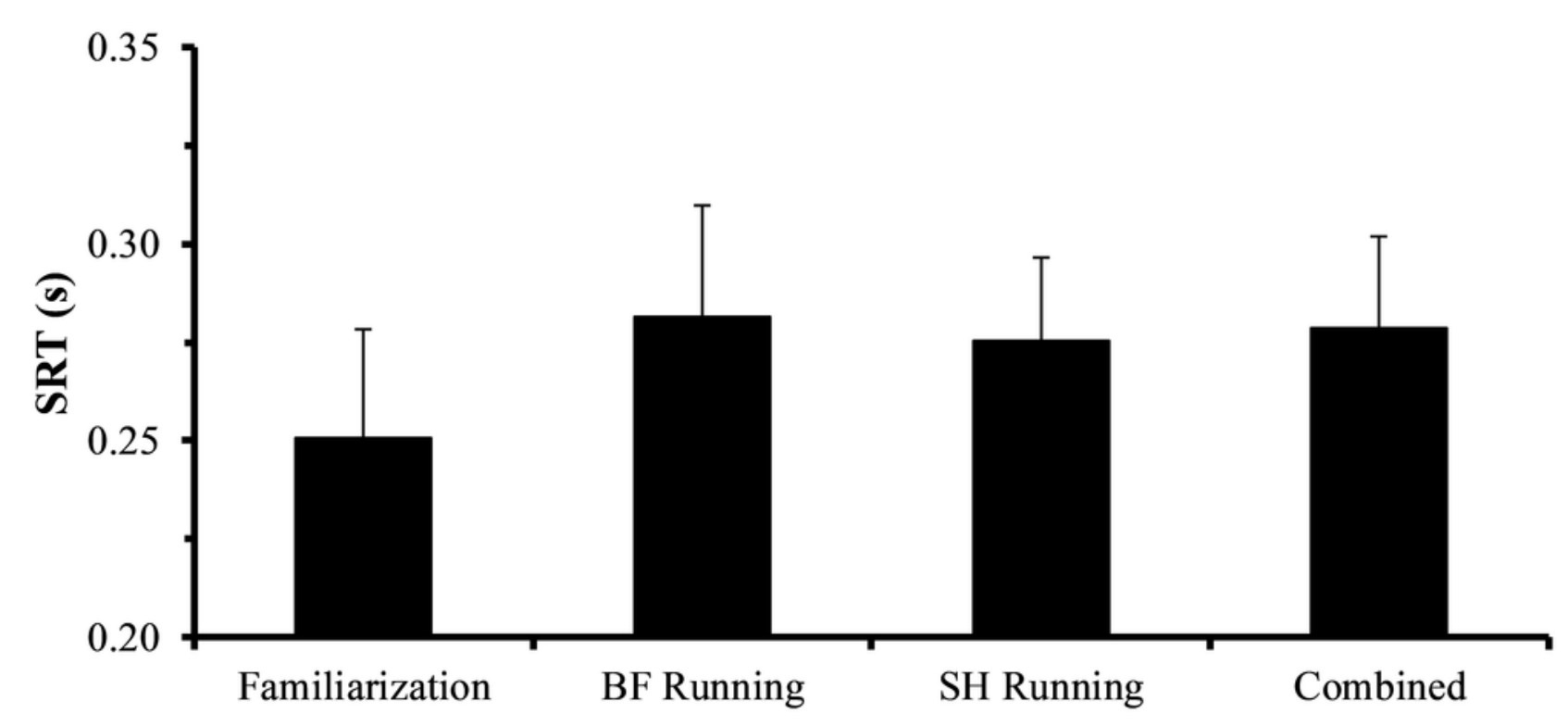

Condition 


\section{Figure 4}

Average SRT errors across barefoot (BF) and shod (SH) running trials.

Average SRT errors across barefoot $(\mathrm{BF})$ and shod $(\mathrm{SH})$ running trials. Absent SRT responses represented $17.6 \%$ and $17.0 \%$ of total SRT trials under the BF and SH running conditions, respectively (17.6 \pm 6.6 trials vs. $17.0 \pm 13.0$ trials). The difference in SRT errors across conditions was no statistically $\left(t_{(7)}=1.07, p=0.918\right)$. Vertical bars represent one SD.

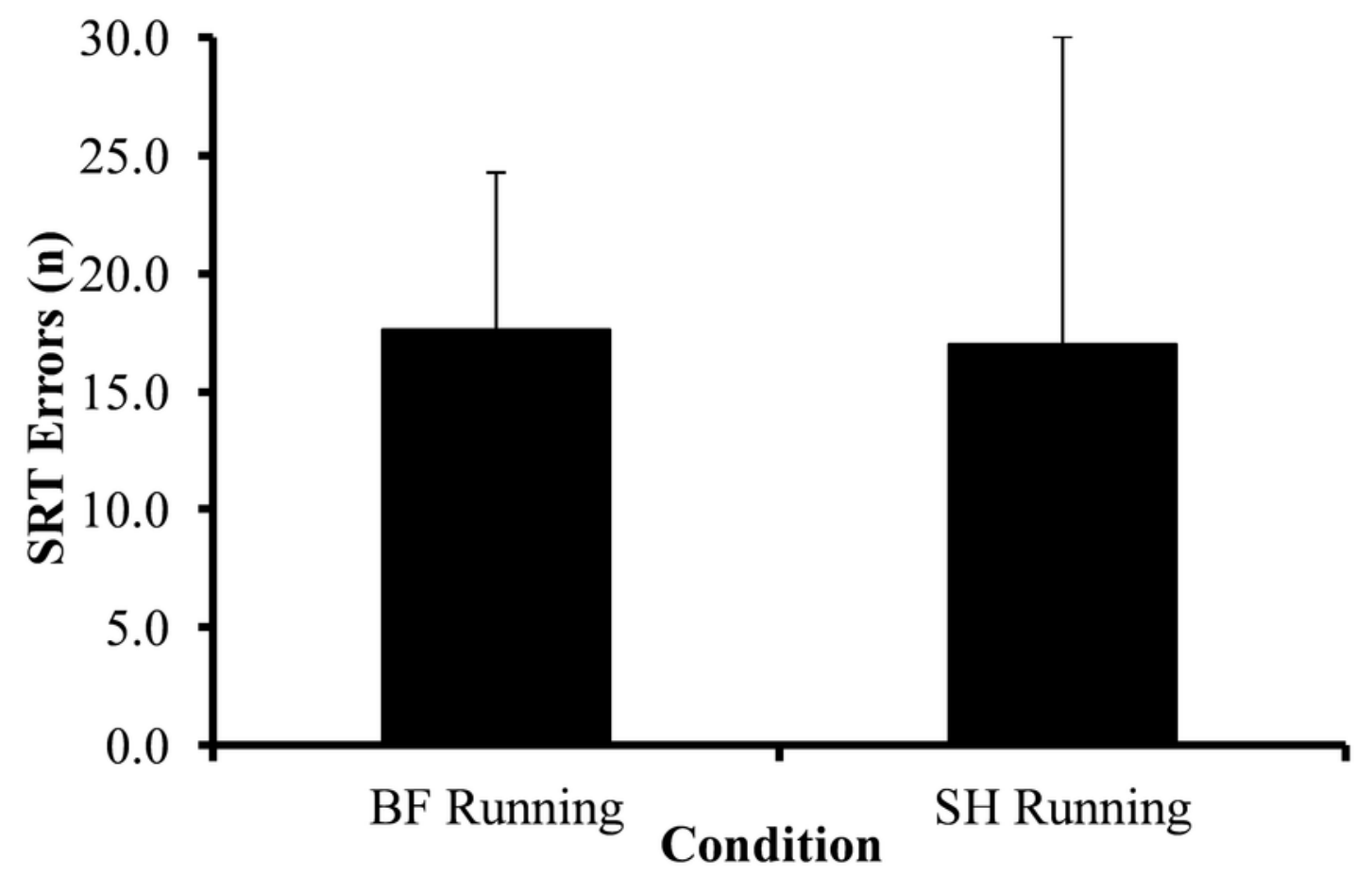


Table $\mathbf{1}$ (on next page)

Participant characteristics (Mean \pm SD). 
1 Table 1. Participant characteristics (Mean \pm SD).

\begin{tabular}{|ccccccc|c|c|}
\hline Age & \multicolumn{1}{c}{ Body Mass } & Height & BMI & $\mathrm{HR}_{\max }$ & \multicolumn{2}{c|}{ \% Age-predicted $\mathrm{HR}_{\max }$} & $\mathrm{VO}_{2 \max }$ & MAS \\
\hline$(\mathrm{yr})$ & $(\mathrm{kg})$ & $(\mathrm{cm})$ & $\left(\mathrm{kg} \mathrm{m}^{-2}\right)$ & $(\mathrm{bpm})$ & $(\%)$ & $\left(\mathrm{mL} \mathrm{min}^{-1} \mathrm{~kg}^{-1}\right)$ & $\left(\mathrm{km} \mathrm{h}^{-1}\right)$ \\
\hline \hline $25.1 \pm 3.7$ & $78.4 \pm 8.9$ & $180.7 \pm 7.8$ & $24.0 \pm 2.0$ & $191 \pm 4$ & $96.5 \pm 6.5$ & $61.4 \pm 6.7$ & $17.9 \pm 1.4$ \\
\hline
\end{tabular}

2 BMI, body mass index; $\mathrm{HR}_{\max }$, maximal heart rate; $\mathrm{VO}_{2 \max }$, maximal oxygen uptake; MAS, maximal aerobic speed. 
Table 2 (on next page)

Participant training profile (Mean $\pm S D$ ). 
1 Table 2. Participant training profile (Mean \pm SD).

\begin{tabular}{|ccccc|c|c|}
\hline $\begin{array}{c}\text { Training } \\
\text { Experience }\end{array}$ & $\begin{array}{c}\text { Training } \\
\text { Sessions }\end{array}$ & $\begin{array}{c}\text { Interval Training } \\
\left(>75 \% \mathrm{VO}_{2 \max }\right)\end{array}$ & $\begin{array}{c}\text { Training } \\
\text { Load }\end{array}$ & $\begin{array}{c}\text { Minimalist Shoe } \\
\text { Experience }\end{array}$ & $\begin{array}{c}\text { Minimalist Training } \\
\text { Load }\end{array}$ & $\begin{array}{c}10 \mathrm{~km} \text { Personal } \\
\text { Best }\end{array}$ \\
\hline$(\mathrm{yr})$ & $\left(\mathrm{n} \mathrm{wk}^{-1}\right)$ & $\left(\mathrm{n} \mathrm{wk}^{-1}\right)$ & $\left(\mathrm{km} \mathrm{wk}^{-1}\right)$ & & $\left(\mathrm{km} \mathrm{wk}^{-1}\right)$ & $(\mathrm{min}: \mathrm{s})$ \\
\hline \hline $3.1 \pm 2.1$ & $8.1 \pm 3.5$ & $1.9 \pm 0.8$ & $90.0 \pm 44.7$ & $11.5 \pm 11.4$ & $72.3 \pm 62.0$ & $37: 26 \pm 2: 50$ \\
\hline
\end{tabular}

$2 \mathrm{VO}_{2 \text { max }}$, maximal oxygen uptake. 